\title{
IMPACT OF COVID 19 ON HEALTH SECTOR
}

KEY WORDS: Pandemic, Medical Sector, Medical System, Health Care Workers and Pharmaceutical

\section{Dr. J. Sujitha Jacinth}

Associate Professor, Department of Anatomy, Rajah Muthiah Medical College Annamalai University, Chidambaram, India.

The Covid 19 outbreak has turned the world upside down by affecting from individual's life style to a country's economy. It doesn't leave medical sector too. The corona outbreak reshaped the medical systems all over the world as this pandemic proved the fragility of medical systems and the necessity for reconstruction of medical sector and to improvise the facilities and care provided to the patients. This article throws light on the impact of Covid - 19 on medical sector. The pandemic has deeply affected the live styles of health care workers. The increase in work pressure, changes in work time, inadequacy of personal protective equipments, higher risk of exposure to infection, treating the stigmatized patients have profoundly increased their stress levels. The fact is that the infection rate is more among the medical professionals than the common public. Hence health care workers are affected physically as well as psychologically due to this pandemic. Being away from family and friends made them to lose their personal connectivity with the society and has affected their social well being. Though pharmaceutical industries are benefitted by the pandemic due to the increased production of aprons, gloves, sanitizers, they are also stressed due to the process of continuous research to discover vaccines to overcome this outbreak. Unlike other sectors, private health providers are having major drawback financially with lesser patients and low number of surgeries than before. Patients with non Covid diseases suffer greatly as their periodic consultations with their physicians were not possible. This caused a dilemma in taking medicines and is unable to know the prognosis of the disease. So the patients are physically affected and also stressed. Thus the impact of Covid 19 on medical industry is that it is going to revolutionize the medical industry thoroughly.

\section{INTRODUCTION}

Pandemicisan epidemic occurring over a very wide area, crossing international boundaries, and is affecting a large number of people. It happens when a novel virus emerges among human-it causes serious illness and is easily human transmissible(1).The word pandemic comes from the Greek 'pandemos' meaning 'pertaining to all people'. The Greek word pan means all and the Greek word demos mean people. Only some pandemics cause severe disease in some individual or at a population level. If immunity is low or nonexistent the spread of infection will be more.

\section{COVID 19}

Covid 19 expanded as the corona virus disease of 2019 is a respiratory illness caused by the novel Corona virus now called Severe Acute Respiratory Syndrome Corona virus2(SARS-COV-2).The disease was first discovered in Wuhan, capital of Hubei province of People's Republic of China on November of 2019 On Jan 30, 2020 WHO declared COVID-19 outbreak, a global health emergency. The Centre for Disease Control and Prevention postulated that this situation would result in a very large number of patients requiring medical care concurrently, resulting in overloaded public healthcare systems, and elevated rates of hospitalizations and deaths and declared as Pandemic by theWHO on 11 th March of 2020.

Since its discovery, the Covid 19 disease has created a sense of worry in people all across the world especially the frontline warriors. It has certainly had many impacts on the economy of many nations by affecting all the sectors of work. But it's effect on the healthcare system is unimaginable. The COVID-19 pandemic has put our health systems under immense pressure and stretched them beyond their capacity. Failure to protect health care in this rapidly changing context exposes health systems to critical gaps in services when they are most needed, and can have a long-lasting impact on the health and wellbeing of populations.

\section{MATERIALS AND METHODS}

The study was conducted among 200 health professionals who include 100 males and 100 females. The health professionals included in the survey are doctors, nurses, and technicians. The questionnaire includes depression, anxiety, stress and effects of wearing PPE.

\section{RESULTS}

$98 \%$ of people experienced anxiety and stress. $2 \%$ health professionals experienced depression. Nurses were affected more than doctors. Gender wise females were more affected than males. $90 \%$ of people experienced the side effects of PPE like rash, erosions over nasal bridge.70\% experienced other symptoms like fever, cough, weakness. $5 \%$ became Covid 19 positive.

\section{DISCUSSION}

Corona virus constitutes the subfamily ortho corona viridae, in the family corona viridae and order nido virales. They are enveloped viruses with a positive sense single stranded RNA genome and a nucleo capsid of helical symmetry. They have characteristic club shaped spikes that project from their surface, which in electron microscope looks like a crown. Corona viruses are currently classified into four genera : alpha coronavirus, beta coronavirus, gamma corona virus and delta corona virus. Among this beta corona virus genera descend from bat gene pool, related to human SARS and MERS. Both alpha and beta infect bats and can infect other species, including humans, camels, rabbits and others. The other two genera primarily infect birds. In human infection, there are mainly six types of recognised coronaviruses namely 229E, NL63 under alpha type and OC43, HKU1, SARSCOV and MERS-COV comes under beta corona virus type(2).Transmission of infection is by coughing, sneezing and personal contact, SARS COV also spreads by droplets and rarely airborne.

In 1920 - Report of corona virus infection in animals, when an acute respiratory infection of domesticated chicken emerged in North America. 1931 - Arthur Schalk and M.C. Hawn made the first detailed report which described a new respiratory infection of chickens in North Dakota.1933 - Leland David Bushnell and Carl Alfred Brandy isolated the virus that caused the infection. This virus then known as 'Infectious bronchitis virus'. In 1937 - Charles D.Hudson and Fred Robert Beaudette cultivated the virus for the first time. The specimen came to be known as Beaudette strain. In1960 - Human corona viruses were discovered.1961 - Isolated the first humancoronavirus strain as 'B814'.1962 - Dorothy Havre and John Procknow at the University of Chicago isolated a new strain as '229E'. 1967 - A research group of National Institute of Health was able to 
isolate another member as 'OC43'. Otherhuman Coronaviruses (HCoV)have since been identified including - SARS-CoV in 2003, HCoV NL63 in 2003, HCoV HKUl in 2004 ,MERS-CoV in 2013 and SARS-CoV2 in 2020. Four human corona viruses produce symptoms that are generally mild namely.HCoVOC43, .HCoV-HKUl,.HCoV-229E,.HCoV-NL63.Three human corona viruses produce symptoms that are generally severe:

- Middle East Respiratory syndrome related coronavirus (MERS-CoV). Outbreaks in 2012,2015,2018.

- Severe acute respiratory syndrome coronavirus (SARSCoV). Outbreaks in 2002,2004.

- Severe acute respiratory syndrome coronavirus 2 (SARSCoV2).2019-2020 pandemic.

Almost all the countries in the world battled the situation of lack of beds and other necessary support for patients with Covid 19. India with its enormous population had battled with many diseases with less number of health care workers. Though it has been a topic of debate for years now, Covid 19 pandemic has unveiled this situation to all the people.This could force the Government to work towards reducing workload of health care workers.Increase in Primary Health Care burden as overcautious people throng general Practitioners even on mild symptoms.

Mental health is important for all sectors of people. A large proportion of Healthcare professionals are experiencing symptoms of anxiety, depression, insomnia, and psychological distress(3). . Nurses often report severe symptoms as they must engage in a lot of emotional labor,like reassuring patients while keeping their own emotions in check.. Grief at the loss of loved ones, anxiety at the loss of jobs, isolation and restrictions on movement, difficult family dynamics, have their impacts on mental health of health care workers. It is reported that some doctors leave the Covid hospital, and join a non Covid facility as they couldn't handle the stress. In treating patients there has to be a 'trust factor'. If the doctors' mental well-being is getting affected then how can they treat the patients. This makes them leave the covid duty.Health care workers deal with life and death of numerous people. In many covid casualties, it is the hospital staff, who have performed the last rites of the patients and have been the last persons to be with patients on their deathbed holding their hands as they gasp for the last few breaths remaining in them. These experiences definitely impact their emotional and mental health.In situations like a pandemic or epidemic where continuous work hours and seeing the pain or death of numerous patients might affect the mental health of the health care workers.Therefore, the need for awareness regarding mental health and normalising seeking professional help for mental health disturbance become a basic necessity for them.Social ostractions, like not allowing doctors and nurses to stay in their home or use the common elevator have added to these stresses.

A study by Natasha Shaukat et al on Physical and mental health impacts of COVID-19 on healthcare workers showed that they commonly experienced high levels of depression, anxiety, insomnia and distress. Anxiety in females was higher than in males, nurses higher than doctors. The study also showed that the most common symptoms identified amongst Healthcare workers were fever (85\%), cough(70\%), and weakness(70\%)(4).According to a study conducted by Lai J et al the impacts are overall anxiety (23-44\%), severe anxiety $(2.17 \%)$, moderate anxiety $(4.78 \%)$, mild anxiety (16.09\%), stress disorder(27.4-71\%), depression (50.4\%), and insomnia(34.0\%)(5) A study published in May 2020, in the 'Indian journal Psychiatry' reveals how dealing with Covid pandemic is taking a toll on the mental health of doctors. Of the 152 doctors who participated in that study, 39.5\% reported experiencing symptoms of anxiety and $34.9 \%$ reported experiencing depression due to longer duty hours, involvement in high risk procedures and washing hands at regular intervals(6). WHO also stated that nurses, midwives and other health workers are at greater risk of COVID-19 than anyone else. According to Indian Medical Association COVID-19 data report on SEP 16, 2238 doctors were infected with the disease and 382 lost their lives. Doctors suffer four times greater mortality than ordinary citizens and private practitioners eight times greater mortality on the same scale.

The pandemic has put pressure on our health system's ability to provide PPEs and compelled to accept PPEs with substandard quality. Due to the PPE material allergic or irritant contact dermatitis may develop. The health workers find very difficult to cope up especially during summer. It also makes verbal communication difficult, loss of sense of smell, reduced tactile sensitivity and reduced vision due to water vapor deposited on goggles. Some procedural errors and unintentional self injuries have also been reported. This has increased the work stress, anxiety and fear of working in lifethreatening environment. Excessive sweating leads to dehydration, fungal infections are also noted. The tight strap of the mask causes skin damage and erosions over nasal bridge due to the mask fit..Due to Hypoxia there may be acute headache, fatigue, exhaustion, giddiness and some even faint. Prolonged PPE usage led to coetaneous manifestations and skin damage $(97 \%)$, with the nasal bridge $(83 \%)$ as the most commonly affected site(4). The cutaneous manifestations are also due to more than 10 times/day hand hygiene. Usage of PPE also causes mental illness as they have to be like that for an average of 6 hours without drinking a drop of water or without using latrines. This suggests, implementation of strategies to reduce the chances of infections and shortern the shift lengths could help the health care workers fighting against covid 19.Risk of infection from the PPE if doffing is not done properly. Donning of PPE takes its own time and until then the patient suffers. For example: if an anesthetist is called forthe intubation and he take 2-3 mins for wearing the PPE, the patient may die within that time. It also unveiled another scenario of lack of Personal protective equipment for all health care workers. Government should consider similar scenarios in future and provide all health care workers with protective equipments.

According to WHO survey June 2020, prevention and treatment services for non-communicable diseases have been severely disrupted since COVID-19 pandemic begun. The survey was completed by 155 countries during 3 week period in May. People living with NCDs are at higher risk of severe COVID-19 related illness. Many people who need treatment for diseases like cancer, cardiovascular diseases and diabetes have not been receiving health services and medicines.

\begin{tabular}{|c|c|}
\hline DISEASE & $\begin{array}{l}\text { PARTIAL OR } \\
\text { COMPLETE } \\
\text { DISRUPTION } \\
\text { OF } \\
\text { TREATMENT } \\
\end{array}$ \\
\hline HYPERTENSION & $53 \%$ \\
\hline DIABETES AND RELATED COMPLICATIONS & $49 \%$ \\
\hline CANCER & $42 \%$ \\
\hline CARDIOVASCULAR EMERGENCIES & $31 \%$ \\
\hline REHABILITATION & $63 \%$ \\
\hline
\end{tabular}

In $94 \%$ of countries, Ministry of health staff working in areas of NCDs are partially or fully reassigned to support covid 19 . This resulted in postponement of screening programmes like breast and cervical cancer. One of the main reasons for discontinuing services was shortage of medicines, diagnostics and other technologies.Elective procedures for nonemergent conditions are difficult to see treatment for.Treatment for diseases that are not covid are getting hit and those who come for that to the hospital expose themselves to infection at the hospital . Restaurants and retailers aren't the only small business that have taken a financial hit during covid 19 pandemic. Physicians in small private practices around the world have reported steep 
declines in their revenues.The Medical Group Management Associations found that covid 19 had a negative effect on $97 \%$ of the 724 medical practices it surveyed. Majority of the small private practicians reduced their working hours half and some had their salaries reduced.

There had been numerous situations where people glorify health care workersbut the Covid 19 pandemic caused a backlash from one group of people who failed to treat health care workers as humans. The pandemic has created hostile environment for all the frontline workers, and there is an increasing report of violence, stigmatization and harassment.There have been numerous similar situations in the past where health care workers have been attacked for unavoidable deaths.Covid 19 pandemic further added to the confusions and has created a feeling of insecurity among health care workers.According to WHO, attack on health care workers is defined as, "any verbal or physical act of violence, obstruction and threat that interferes availability, access and delivery of health services."According to the surveillance system for attacks on health care (SSAPhysical assault.) types of reported attacks related to COVID 19 are denial of services. psychological threat,.Heavy weapon.Obstruction,Eviction from home.This has also affected psychological health of the patients, health care providers and their families. There is a shortage of health care workers since many are unwilling or unable to report to work because of unsafe environments. This validates the need for proper education regarding consent and medical practices among common people and pass laws that ensure safety of health care workers . Protecting the health and lives of frontline workers is crucial for better global response.

The expectations of the public and government from the pharmaceutical industries has tremendously increased in the pandemic. As the masks like protective measures become a huge necessity, they are expected to produce and deliver these as soon than earlier.The pharmaceutical industries are highly benefitted by increase in production of personal protective equipments, pulse oximeter, aprons, gloves, sanitizers etc which made a huge profit for them. On the other side sale of surgery related drugs like anaesthetic agents, instruments, etc. have gone down. Neccessity of vaccines to overcome this outbreak majorly rely on contributions of pharmaceutical industrial researches. Besides the protective kits, there is a huge pressure on pharmaceutical research for discovering the vaccine. Even if the vaccines are discovered, it will create a pharmaceutical revolution of high benefit. Situation in China has disrupted the exports of critical raw materials and medical devices .this has put upward pressure on prices of Medical devices in short term. COVID 19 crisis has again highlighted the contribution of Indian pharma industry as India opened export of hydroxychloroquine to scores of countries that can help save thousands of lives.

Health insurance policies have gone up Covid 19 pandemic had made people aware of the potential risk of infection health care workers face in a hospital environnemt. Understanding the severity of the pandemic, Government and other Insurance companies came forward with schemes for health care workers at the time of Covid 19 pandemic. But health care workers battle with more diseases other than Covid 19.Therefore, the government should come up with an insurance scheme by the government for health care workers at all times even after Covid 19.

Fast tracking of implementation of targets for public health emergencies within NATIONAL DISASTER MANAGEMENT PLAN is formulated. Indian government has planned to increase $2.5 \%$ GDP to strengthen government infrastructure and public private partnerships over next few years...Medical tourism has seen a drop significantly. Affordability of medical treatment in India has made the country a desirable alternatives to other countries like Western Europe, North
America and some South East Asian countries. India is quickly becoming the premier destination for foreigners seeking the same quality treatments at a much cheaper price with less investment on transportation and living costs. According to report by Federation of Indian chambers of Commerce and Industrty (FICCI) and IMS Health India has nearly 18\% of global medical tourism market. India has ranked $5^{\text {th }}$ position among 41 major medical tourism destinations as per Medical Tourism Index Overall ranking ,2016.India has ranked $2^{\text {nd }}$ in Asia as per the international healthcare and research centre statistics. It has also been estimated that Indias medical tourism industry account for $20 \%$ of global market share by 2020.The probable reason is the availability of both modern medicine and ayush sector like Ayurveda ,Unnani and Naturopathy. But now Indias medical tourism industry has faced a massive blow due to ban on international travel. Investors are not ready to invest in startups of medical tourism space.

Due to advancement in technology, Telemedicine serves an effective way to alleviate the congestion of hospitals especially during such a pandemic and improves the utilization of medical resources. The goal of telemedicine is to deliver health care to patients in remote and rural areas in order to overcome the problems like shortage of medical care professionals and health care facilities. This has also been extended to people leading busy schedules and lifestyles. People are nowadays not willing to waste their time in travelling to the clinic and waiting in line.. .according to national rural health association (NHRA) there are only 30 specialists available for every 1 lakh people in rural areas. Medical issues can be quickly addressed through real time consultations within 30 minutes. However it may not be possible for all people to access telemedicine. Protecting the medical data of patients may be a problem with hackers having access to the data. Emergency treatments may become a troublesome problem. Licensing issues, technical concerns and inability to examine the patients are major issues in urban areas turning to tele health has happening now. A change that would have expected over a decade happened in a month. Telemedicine has helped make up for some of the decline in office visits. Telemedicine Practice Guidelines enabling registered Medical Practitioners to provide healthcare in remote settings.

All personnel in the Frontline should be made aware of the principles of self care. In order to reduce stress and depression they should have to ensure breaks and adequate sleep, Keep in touch with relatives and friends, Carry out some activities and hobbies unrelated to work Exercise regularly and have a healthy diet, Practice yoga, Listening to music. Increased use of technology, telemedicine, training of primary health workers and mobile hospitals can be seen in foreseeable future of health sector.

\section{CONCLUSION}

According to Hippocrates, the father of medicine, health is the greatest of human blessings, stated by the with the guidance of great people like him, each and every day health sector is moving to an entirely different level of providing health care. The pandemic has increased the momentum of these changes. From being a stressful pandemic to evolutionary pandemic ,The Covid 19 pandemic has highlighted issues like lack of healthcare facilities for the people, lack of personal protective equipment for the health care workers, violence against health care professionals and risks faced by the health care workers physically and mentally. It has validated the need to take measures to safeguard the health care workers physically, mentally and socially. Community awareness towards hygiene will have positive impact in long term.

\section{REFERENCES}

1. Apurba Sastry \& Sandhya Bhat (2019). Essentials of Medical Microbiology, $2^{\text {nd }}$ 
2. Cullion.Jay Pee Publishers (542-543).

Pandemic.QJM.;113(5):311-312.doi:10.1093/qjmed/hcaallo.

3. Park. K. (2019). Parks Textbook of Preventive and Social Medicine, $25^{\text {th }}$ edition. Banarsidas Bhanot Publishers(101 \& 109).

4. Lai J, Ma S, Wang Y, Cai Z, Hu J, Wei N, et al(2019). Factors associated with mental health outcomes among health care workers exposed to coronavirus disease.JAMA New Open3(3):e203976.

5. Natasha Shaukat, Daniyal Mansoor Ali, Junaid Razzak(2020). Physical and mental impacts of Covid -19 on healthcare workers: a scoping review. In the J Med.;13:40.doi:0.1186/s12245-020-00299-5.

6. Seshadri Sekhar Chatterjee, Ranjan Bhattacharyya, Sumita Bhattachaaryya Soumitra Das,Bejoy Bikram Banerjee(2020). Indian J.Psychiatry 3(62):257265,20200501 . 